\title{
Global Uniform Asymptotic Stability of a Class of Switched Linear Systems with an Infinite Number of Subsystems
}

\author{
L. F. Araghi, ${ }^{1}$ A. A. Suratgar, ${ }^{2,3}$ and E. Feizi ${ }^{4}$ \\ ${ }^{1}$ Department of Electrical and Computer Engineering, Science and Research Branch, Islamic Azad University, \\ Tehran 14515-775, Iran \\ ${ }^{2}$ Department of Electrical Engineering, Amirkabir University of Technology, Tehran, Iran \\ ${ }^{3}$ The Center of Excellence on Control and Robotics, Department of Electrical Engineering, Amirkabir University of Technology, \\ Tehran, Iran \\ ${ }^{4}$ Department of Mathematics, Bu-Ali Sina University, Hamedan, Iran
}

Correspondence should be addressed to L. F. Araghi; 1.fallah@srbiau.ac.ir

Received 31 December 2013; Revised 9 March 2014; Accepted 28 March 2014; Published 19 May 2014

Academic Editor: Ryan Loxton

Copyright (c) 2014 L. F. Araghi et al. This is an open access article distributed under the Creative Commons Attribution License, which permits unrestricted use, distribution, and reproduction in any medium, provided the original work is properly cited.

\begin{abstract}
Stability of switching systems with an infinite number of subsystems is important in some structure of systems, like fuzzy systems, neural networks, and so forth. Because of the relationship between stability of a set of matrices and switching systems, this paper first studies the stability of a set of matrices, then and the results are applied for stability of switching systems. Some new conditions for globally uniformly asymptotically stability (GUAS) of discrete-time switched linear systems with an infinite number of subsystems are proposed. The paper considers some examples and simulation results.
\end{abstract}

\section{Introduction}

A switched system is a dynamical system that consists of a finite or infinite number of subsystems and a logical rule that orchestrates switching between these subsystems. Mathematically, these subsystems are usually described by a collection of indexed differential or difference equations. One convenient way to classify switched systems is based on the dynamics of their subsystems, for example, continuoustime or discrete-time, linear or nonlinear, and so on [1]. In the recent years, many researchers have investigated on the stability of switching systems. Lin and Antsaklis proposed a necessary and sufficient condition for asymptotic stabilization of switched linear systems [1]. In [2] absolute asymptotic stability of discrete linear inclusions in Banach (both finite and infinite dimensional) space was studied and the relation between absolute asymptotic stability, asymptotic stability, uniform asymptotic stability, and uniform exponential stability was established.

The concept of state-norm estimators for switched nonlinear systems under averaged well-time switching signals was studied in [3]. Liberzon and Trenn showed that switching between stable subsystems may lead to instability and that the presence of algebraic constraints leads to a larger variety of possible instability mechanisms [4].

Some new sufficient conditions for exponential stability of switched linear systems under arbitrary switching were proposed in [5]. Hien et al. considered the problem of exponential stability and stabilization of switched linear timedelay systems. They assumed system parameter uncertainties are time varying and unknown but norm bounded [6].

A new sufficient condition that guarantees global exponential stability of switched linear systems based on Lyapunov-Metzler inequalities was proposed in [7]. A class of uncertain impulsive systems with delayed input was studied in [8]. It was shown that these systems can be transformed into switched systems without time delay.

Hante and Sigalotti considered switched systems on Banach and Hilbert spaces governed by strongly continuous one-parameter semigroups of linear evolution operators. They provided necessary and sufficient conditions for their global exponential stability, uniform with respect to the 
switching signal, in terms of the existence of a Lyapunov function common to all modes [9].

$\mathrm{Du}$ et al. proposed finite-time stability and stabilization problems for switched linear systems. Firstly, they extended the concept of finite-time stability to switched linear systems. Then, a necessary and sufficient condition for finite-time stability of switched linear systems was presented based on the state transition matrix of the system [10]. Stability and $L_{2}$ gain properties for a class of switched systems which are composed of normal discrete-time subsystems were studied in [11]. It was shown that when all subsystems are Schur stable, $V(x)=x^{T} x$ is a common quadratic Lyapunov function for the subsystems and the switched normal system is exponentially stable under arbitrary switching.

The exponential stability and stabilization problems of a class of nonlinear impulsive switched systems with timevarying disturbances were studied in [12], and some sufficient conditions were obtained by using the switched Lyapunov function method as algebraic inequality constraints and linear matrix inequalities.

Santarelli studied switched state feedback control law for the stabilization of LTI systems of arbitrary dimension [13]. A unified approach to stability analysis for switched linear descriptor systems under arbitrary switching in both continuous-time and discrete-time domains was studied in [14]. The proposed approach was based on common quadratic Lyapunov functions incorporated with linear matrix inequalities (LMIs). The boundedness and attractiveness of nonlinear switched delay systems whose subsystems have different equilibria were studied in [15]. It was shown that the nonlinear switched delay system is attractive and obtained the attractive region. The authors of the paper proposed three methods for existence of a common quadratic Lyapunov function for robust stability analysis of fuzzy Elman neural network [16].

Since switched systems are used in many applications such as robotics, integrated circuit design, manufacturing, power electronics, switched-capacitor networks, chaos generators, and automated highway systems, some applications for linear or nonlinear switched systems have been further investigated, and many valuable results and simulations have been obtained; see [17-20] and some references therein.

In [17], an optimal control problem with dynamics that switch between several subsystems of nonlinear differential equations was considered. It was shown that an approximate solution for this optimal control problem can be computed by solving a sequence of conventional dynamic optimization problems.

An optimal control problem was considered in which the control takes values from a discrete set, the state and control are subject to continuous inequality constraints, and a new computational method was proposed for solving optimal discrete-valued control problems [18].

In [19] an approach with an existing hybrid power system model was explained. The problem of choosing an operating schedule to minimize generator, battery, and switching costs was first posed as a mixed discrete dynamic optimization problem. Some of the latest computational techniques for generating optimal control laws for switched systems with nonlinear dynamics and continuous inequality constraints were reviewed in [20].

In $[21,22]$, some theorems for stability analysis of TSK and linguistic fuzzy models were proposed by the authors.

In the recent years, there are many articles about switched systems and stability of them with a finite number of switching but there is no research about the stability of switched systems when the number of subsystems is infinite. Since the study of this class of switching systems is important in some structure of systems, like fuzzy systems, and so forth, so in this paper, stability analysis of discrete-time switched linear systems with an infinite number of switching is investigated and some new conditions are proposed for globally uniformly asymptotically stability (GUAS) of these systems.

The paper is organized as follows.

Section 2 introduces basic definitions and concepts. In Section 3, we derive some conditions for convergence to zero of infinite product of matrices. In Section 4, we extract some new methods for stability analysis of linear switching systems with infinite number of subsystems and provide some illustrative examples. Section 5 gives conclusions.

\section{Problem Statement}

Consider a set $\Sigma$ of matrices $A_{i_{k}}$ and pick an initial point $x_{0}$, at $t=0$. A switched linear system is a dynamical system of the type:

$$
x_{k+1}=A_{i_{k}} x_{k}, \quad A_{i_{k}} \in \Sigma, i_{k} \in I, k \in \mathbb{Z}^{+},
$$

where $I$ is an infinite index set, $i_{k}$ is switching law, and the state $x \in \mathbb{R}^{n}$ and $A_{i_{k}} \in \mathbb{R}^{n \times n}$ for all $i_{k} \in I[23,24]$.

This note means that, at every instant, the matrix $A_{i_{k}}$ defined to evolve the system can be replaced by another one from the set $\Sigma$.

The stability of a switching system when there is no restriction on the switching signals is usually called stability analysis under arbitrary switching. For this analysis, it is necessary that all the subsystems are asymptotically stable. However, even when all the subsystems of a switching system are exponentially stable, it is still possible to construct a divergent trajectory from any initial condition. So, in general, the assumption of subsystems' stability is not sufficient to ensure stability of switching systems under arbitrary switching, except for some special cases, such as pairwise commutative systems and symmetric or normal systems (all subsystems). Consequently, if there exists a common Lyapunov function for all the subsystems, then the stability of the switching system is guaranteed under arbitrary switching [23].

Definition 1. The linear switching system (1) is globally uniformly asymptotically stable (GUAS) if for any initial condition $x_{0} \in \mathbb{R}^{n}$ and any switching law $i_{k}[23,25]$ :

$$
\lim _{k \rightarrow \infty} x_{k}=0, \quad \forall\left(i_{k}\right)
$$


As this is supposed to hold for any initial vector $x_{0}$, it is equivalent to saying that all matrix products taken from $\Sigma$ converge to the zero matrix; that is,

$$
\lim _{k \rightarrow \infty} A_{i_{k}} A_{i_{k-1}} \cdots A_{i_{1}}=0, \quad \forall\left(i_{k}\right) .
$$

The GUAS problem is closely related to determining the joint spectral radius (JSR) of the set of matrices $\Sigma=$ $\left\{A_{1}, \ldots, A_{n}\right\}$, denoted by $\rho(\Sigma)[26]$.

The joint spectral radius characterizes the maximal asymptotic growth rate of a point submitted to a switching linear system in discrete time. On the other hand, it characterizes the maximal asymptotic growth rate of the norms of long products of matrices taken in as a set $\Sigma$. The stability of the system is ensured when the growth rate is less than one $[25,27]$.

Let $\|\cdot\|: \mathbb{R}^{n} \rightarrow \mathbb{R}_{+}$denote the Euclidean vector norm and denote [27]

$$
\rho_{k}(\Sigma)=\max \left\{\left\|A_{i_{1}} A_{i_{2}} \cdots A_{i_{k}}\right\|^{1 / k}, i_{j} \in\{0,1, \ldots, n\}\right\},
$$

and then the joint spectral radius is defined as

$$
\rho(\Sigma)=\lim _{k \rightarrow \infty} \rho_{k}(\Sigma) .
$$
[26].

The switching system (1) is GUAS if and only if $\rho(\Sigma)<1$

Some results show that computing or even approximating the JSR is extremely hard [25]. Here it is proposed some matrix structures for the subsystems in which the stability of a switching system is guaranteed.

Consider the discrete-time switching linear system as follows:

$$
x(k+1)=\Sigma_{k} x(k), \quad k=1,2, \ldots,
$$

where $\Sigma_{k} \in \Sigma, \Sigma=\left\{\Sigma_{1}, \Sigma_{2}, \ldots\right\}$.

A switching dynamical system of the form (6) is stable if for any initial condition $x_{0} \in \mathbb{R}^{n}$ and any sequence of matrices $\Sigma^{k} \triangleq\left\{\Sigma_{1} \Sigma_{2} \cdots \Sigma_{k}\right\}, \lim _{k \rightarrow \infty} x(k)=0$ [25].

Lemma 2. Let $\left\{A_{k}\right\}_{k \in N}$ be a set of square matrices. If there exists $\alpha<1$ such that for all $k \in N,\left\|A_{k}\right\| \leq \alpha$, then

$$
\lim _{k \rightarrow \infty} A_{k} A_{k-1} \cdots A_{1}=0 .
$$

Proof. From the submultiplicity property of norms,

$$
\|A B\| \leq\|A\|\|B\|
$$

the following equation is correct:

$$
\left\|A_{k} A_{k-1} \cdots A_{1}\right\| \leq\left\|A_{k}\right\|\left\|A_{k-1}\right\| \cdots\left\|A_{1}\right\| .
$$

Since, for all $k \in \mathbb{N},\left\|A_{k}\right\| \leq \alpha$, therefore,

$$
\lim _{k \rightarrow \infty}\left\|A_{k}\right\|\left\|A_{k-1}\right\| \cdots\left\|A_{1}\right\| \leq \lim _{k \rightarrow \infty} \alpha^{k} \longrightarrow 0 .
$$

So,

$$
\lim _{k \rightarrow \infty}\left\|A_{k} A_{k-1} \cdots A_{1}\right\| \longrightarrow 0
$$

by continuity of the norms, it is extracted that

$$
\lim _{k \rightarrow \infty} A_{k} A_{k-1} \cdots A_{1} \longrightarrow 0 .
$$

The next proof is based on the geometric series. It proves the sufficient condition for invertibility of matrices. The space of $n \times n$-matrices with real or complex numbers as elements is considered.

Lemma 3. Let $A$ be a real or complex $n \times n$-matrix and let $I$ be the identity matrix. If $\|I-A\|<1$, then $A$ is invertible.

Proof. Let $B=I-A, A \in \mathbb{M}_{n \times n}(\mathbb{C})$, and $\|B\|=\gamma<1$.

Since $\left\|B^{n}\right\| \leq\|B\|^{n}=\gamma^{n}, \sum_{n=0}^{\infty}\left\|B^{n}\right\| \leq \sum_{n=0}^{\infty} \gamma^{n}<\infty$, hence, $C=\sum_{n=0}^{\infty} B^{n}$ converges in $\mathbb{M}_{n \times n}(\mathbb{C})$.

If $C_{n}=I+B+B^{2}+\cdots+B^{n}$, then

$$
\begin{aligned}
C_{n}(I-B)= & \left(I+B+B^{2}+\cdots+B^{n}\right) \\
& -\left(B+B^{2}+\cdots+B^{n+1}\right) \\
= & I-B^{n+1},
\end{aligned}
$$

whereas

$$
\left\|B^{n+1}\right\| \leq \gamma^{n+1}, \quad \text { so } B^{n+1} \longrightarrow 0 \text { as } n \longrightarrow \infty .
$$

As a result,

$$
C(I-B)=\lim C_{n}(I-B)=I .
$$

Similarly, $(I-B) C=I$.

So $(I-B)$ is invertible and $(I-B)^{-1}=\sum_{n=0}^{\infty} B^{n}$.

Finally, $I-B=I-(I-A)=A$ (for more details see [28]).

\section{Infinite Product of Matrices}

In this section some conditions are studied for convergence to zero of infinite product of matrices.

Theorem 4. Let $A_{1}, A_{2}, \ldots$ be a sequence of $n \times n$ matrices and $\|\cdot\|$ a matrix norm. If the sequence satisfies (1) and (2):

(1) $\sum_{k=1}^{\infty}\left(\left\|A_{k}\right\|_{+}-1\right)$ converges,

(2) $\sum_{k=1}^{\infty}\left(1-\left\|A_{k}\right\|_{-}\right)$diverges,

where $\|A\|_{+}=\max \{\|A\|, 1\},\|A\|_{-}=\min \{\|A\|, 1\}$, and $A_{i_{1}}, A_{i_{2}}, \ldots$ is any rearrangement of the sequence, then $\prod_{k=1}^{\infty} A_{i_{k}}=0$.

Thus, this sequence converges to 0 , and so $A_{i_{1}}, A_{i_{2}} A_{i_{1}}, \ldots$ converges to 0 [27].

Theorem 5. Let $\Sigma$ be a compact matrix set as $\Sigma=\left\{\Sigma_{1}, \Sigma_{2}, \ldots\right\}$. Then every infinite product taken from $\Sigma$ converges to 0 iff $\hat{\rho}(\Sigma)<1$, where $\hat{\rho}(\Sigma)$ is the joint spectral radius of a set of matrices [27].

Corollary 6. Let $\Sigma$ be a compact matrix set. Then every infinite product, taken from $\Sigma$, converges to 0 iff there is a norm $\|\cdot\|$ such that $\|A\| \leq \gamma, \gamma<1$, for all $A \in \Sigma[27]$. 
The next theorem refers to a specific structure of matrices.

Consider the discrete-time switching linear system (6) such that

$$
\Sigma_{k}=\left[\begin{array}{cc}
A_{k} & B_{k} \\
0 & C_{k}
\end{array}\right]
$$

where $\Sigma_{k} \in \Sigma, \Sigma=\left\{\Sigma_{1}, \Sigma_{2}, \ldots\right\}$ and $k$ is an infinite index set, $k=1,2, \ldots . .\left[A_{k}\right] \in R^{n_{1} \times n_{2}},\left[B_{k}\right] \in R^{n_{1} \times n_{2}}$ and $\left[C_{k}\right] \in R^{n_{2} \times n_{2}}$, and the state $x \in \mathbb{R}^{n}, \Sigma_{k} \in \mathbb{R}^{n \times n}$.

Theorem 7. Let $\left(\Sigma_{k}\right)_{k \in \mathbb{N}}$ be a sequence of Matrices of the form (16) and let there exist two numbers $\alpha$ and $\gamma$ such that $\gamma<1, \alpha<1$, and $\left\|A_{i}\right\| \leq \alpha,\left\|C_{i}\right\| \leq \gamma$ for some matrix norm $\|\cdot\|$. The sequence $P_{k}=\Sigma_{1} \Sigma_{2} \cdots \Sigma_{k}$ (infinite product of matrices) converges to zero if and only if $A_{1} A_{2} \cdots A_{k-1} B_{k}\left(I-C_{k}\right)^{-1}$ converges to zero.

Proof. To prove the sufficient condition, by construction $P_{k}$ from $\Sigma_{k}$ as follows:

$$
\begin{aligned}
& P_{k}=\Sigma_{1} \Sigma_{2} \cdots \Sigma_{k}, \quad \Sigma_{k} \in \Sigma, k \in \mathbb{N}, \\
& P_{k}=\left[\begin{array}{cc}
A_{1} A_{2} \cdots A_{k} & X_{k} \\
0 & C_{1} C_{2} \cdots C_{k}
\end{array}\right],
\end{aligned}
$$

where

$$
\begin{aligned}
X_{k}= & A_{1} A_{2} \cdots A_{k-1} B_{k}+A_{1} A_{2} \cdots A_{k-2} B_{k-1} C_{k} \\
& +A_{1} A_{2} \cdots A_{k-3} B_{k-2} C_{k-1} C_{k}+\cdots .
\end{aligned}
$$

By hypothesis of the theorem and Lemma 2 , since $\left\|A_{i}\right\| \leq$ $\alpha<1,\left\|C_{i}\right\| \leq \gamma<1$, where $i \in \mathbb{N}$,

$$
\lim _{k \rightarrow \infty} A_{1} A_{2} \cdots A_{k}=\lim _{k \rightarrow \infty} C_{1} C_{2} \cdots C_{k}=0 .
$$
and so

Therefore $\lim _{k \rightarrow \infty} P_{k}=0$ implies that $\lim _{k \rightarrow \infty} X_{k} \rightarrow 0$

$$
\lim _{k \rightarrow \infty} X_{k}-X_{k-1}=0
$$

By some calculations, between $X_{k}$ and $X_{k-1}$, the following relation is achieved:

$$
X_{k}=X_{k-1} C_{k}+A_{1} A_{2} \cdots A_{k-1} B_{k}, \quad(k \in \mathbb{N}) .
$$

By subtraction $X_{k-1}$, the above equation is

$$
\begin{aligned}
X_{k}-X_{k-1} & =X_{k-1} C_{k}+A_{1} A_{2} \cdots A_{k-1} B_{k}-X_{k-1} \\
& =X_{k-1}\left(C_{k}-I\right)+A_{1} A_{2} \cdots A_{k-1} B_{k} .
\end{aligned}
$$

According to Lemma 3 and because of $\left\|C_{i}\right\| \leq \gamma<1$, clearly, $\left(C_{k}-I\right)$ is invertible. Therefore,

$$
\begin{aligned}
& \left(X_{k}-X_{k-1}\right)\left(C_{k}-I\right)^{-1} \\
& \quad=X_{k-1}+A_{1} A_{2} \cdots A_{k-1} B_{k}\left(C_{k}-I\right)^{-1}, \\
& \left(X_{k}-X_{k-1}\right)\left(I-C_{k}\right)^{-1} \\
& \quad=A_{1} A_{2} \cdots A_{k-1} B_{k}\left(I-C_{k}\right)^{-1}-X_{k-1} .
\end{aligned}
$$

From (20) and (23),

$$
\lim _{k \rightarrow \infty} X_{k}=\lim _{k \rightarrow \infty} A_{1} A_{2} \cdots A_{k-1} B_{k}\left(I-C_{k}\right)^{-1},
$$

and consequently

$$
\lim _{k \rightarrow \infty} P_{k}=\left[\begin{array}{cc}
0 & \lim _{k \rightarrow \infty} A_{1} A_{2} \cdots A_{k-1} B_{k}\left(I-C_{k}\right)^{-1} \\
0 & 0
\end{array}\right] .
$$

Namely, by considering the assumed conditions of the Theorem 7, $P_{k}$ converges to zero if $A_{1} A_{2} \cdots A_{k-1}$ $B_{k}\left(I-C_{k}\right)^{-1}$ converges to zero.

To prove the necessary condition of the theorem, it must be verified that if $\lim _{k \rightarrow \infty} A_{1} A_{2} \cdots A_{k-1} B_{k}\left(I-C_{k}\right)^{-1}$ converges to zero, then $\lim _{k \rightarrow \infty} P_{k}=0$.

To prove this, first it must be proved that

$$
\lim _{k \rightarrow \infty}\left\|X_{k}\right\|=\lim _{k \rightarrow \infty}\left\|A_{1} A_{2} \cdots A_{k-1} B_{k}\left(I-C_{k}\right)^{-1}\right\| .
$$

By defining $D_{k}$ as the difference between $X_{k}$ in (18) and $A_{1} A_{2} \cdots A_{k-1} B_{k}\left(I-C_{k}\right)^{-1}$,

$$
D_{k}=X_{k}-A_{1} A_{2} \cdots A_{k-1} B_{k}\left(I-C_{k}\right)^{-1} ; \quad k \in \mathbb{N}
$$

and $Y_{k}$ as

$$
\begin{aligned}
Y_{k}= & A_{1} A_{2} \cdots A_{k} B_{k+1}\left(I-C_{k+1}\right)^{-1} \\
& -A_{1} A_{2} \cdots A_{k-1} B_{k}\left(I-C_{k}\right)^{-1} .
\end{aligned}
$$

From (27) and (28) it is obtained that

$$
\begin{aligned}
D_{k+1} & =X_{k+1}-A_{1} A_{2} \cdots A_{k} B_{k+1}\left(I-C_{k+1}\right)^{-1} \\
& =\left(D_{k}-Y_{k}\right) C_{k+1} .
\end{aligned}
$$

So, $\left\|D_{k+1}\right\| \leq\left(\left\|D_{k}\right\|+\left\|Y_{k}\right\|\right)\left\|C_{k+1}\right\|$, such that $\left\|C_{i}\right\| \leq \gamma$, and therefore,

$$
\begin{gathered}
\left\|D_{k+1}\right\| \leq\left(\left\|D_{k}\right\|+\left\|Y_{k}\right\|\right) \gamma, \\
\left\|D_{k}\right\| \leq\left(\left\|D_{k-1}\right\|+\left\|Y_{k-1}\right\|\right) \gamma, \\
\left\|D_{k-i}\right\| \leq\left(\left\|D_{k-i-1}\right\|+\left\|Y_{k-i-1}\right\|\right) \gamma .
\end{gathered}
$$

By repeating the above inequalities it is obtained that

$$
\begin{gathered}
\left\|D_{k}\right\| \leq\left\|D_{k-i}\right\| \gamma^{i}+\left\|Y_{k-i}\right\| \gamma^{i}+\left\|Y_{k-i+1}\right\| \gamma^{i-1}+\cdots+\left\|Y_{k-1}\right\| \gamma^{1}, \\
\left\|D_{k}\right\| \leq\left\|D_{1}\right\| \gamma^{k-1}+\sum_{i=1}^{k-1}\left\|Y_{k-i}\right\| \gamma^{i}, \quad i=1,2, \ldots, k-1 .
\end{gathered}
$$

Therefore,

$$
\lim _{k \rightarrow \infty}\left\|D_{k}\right\| \leq \lim _{k \rightarrow \infty} \sum_{i=1}^{k-1}\left\|Y_{k-i}\right\| \gamma^{i}, \quad 0 \leq \gamma<1,
$$

because of $\lim _{k \rightarrow \infty}\left\|D_{1}\right\| \gamma^{k-1}=0$. 
By considering $S=\lim _{\sup _{k \rightarrow \infty}}\left\|D_{k}\right\|<\infty$ and since $\lim _{k \rightarrow \infty}\left\|Y_{k}\right\|=0$, so sequence $\left\{\left\|Y_{k}\right\|\right\}_{k=1}^{\infty}$ is bounded; that is, there exist $M>0$ such that for all $k,\left\|Y_{k}\right\| \leq M$. Moreover $\sum_{i=1}^{k-1}\left\|Y_{k-i}\right\| \gamma^{i} \leq M \sum_{i=1}^{k-1} \gamma^{i} \leq M \gamma /(1-\gamma)$ for all $k$.

Thus the sequence $\left\{\sum_{i=1}^{k-1}\left\|Y_{k-i}\right\| \gamma^{i}\right\}_{k=1}^{\infty}$ is bounded. So there exist $S \in \mathbb{R}$ such that $\lim _{\sup _{k \rightarrow \infty}} \sum_{i=1}^{k-1}\left\|Y_{k-i}\right\| \gamma^{i}=S$.

Now, let

$$
\begin{aligned}
S_{k+1} & =\sum_{i=1}^{k}\left\|Y_{k+1-i}\right\| \gamma^{i}=\left\|Y_{k}\right\| \gamma+\sum_{i=2}^{k}\left\|Y_{k+1-i}\right\| \gamma^{i} \\
& =\left\|Y_{k}\right\| \gamma+\gamma \sum_{i=1}^{k-1}\left\|Y_{k-i}\right\| \gamma^{i}=\left\|Y_{k}\right\| \gamma+\gamma S_{k} .
\end{aligned}
$$

In fact, we have $S_{k+1}=\left\|Y_{k}\right\| \gamma+\gamma S_{k}$. By taking the limit superior of both sides of the above equation, it is obtained that $\lim \sup _{k \rightarrow \infty} S_{k+1}=\gamma \lim \sup _{k \rightarrow \infty} S_{k}$, hence $S=\gamma S$, and since $\gamma \neq 1$, then it implies that $S=0$. (27),

The above equation means that $\lim _{k \rightarrow \infty} D_{k}=0$ and from

$$
\lim _{k \rightarrow \infty} X_{k}=\lim _{k \rightarrow \infty} A_{1} A_{2} \cdots A_{k-1} B_{k}\left(I-C_{k}\right)^{-1}, \quad k \in \mathbb{N} .
$$

So, if $\lim _{k \rightarrow \infty}\left\|A_{1} A_{2} \cdots A_{k-1} B_{k}\left(I-C_{k}\right)^{-1}\right\|=0$, then $\lim _{k \rightarrow \infty}\left\|X_{k}\right\|=0$, and also by hypothesis of Theorem 7 and Lemma 2,

$$
\lim _{k \rightarrow \infty} A_{1} A_{2} \cdots A_{k}=\lim _{k \rightarrow \infty} C_{1} C_{2} \cdots C_{k}=0 .
$$

Therefore,

$$
\lim _{k \rightarrow \infty} P=0 .
$$

So it completes the proof.

\section{Stability of Switching Linear Systems with Infinite Number of Subsystems}

The following conditions are extracted for stability of a switching linear system when it comprises an infinite number of subsystems. They are reached from the conditions for convergence to zero of infinite product of matrices.

Corollary 8. The switching linear system (6) is GUAS if the following conditions are satisfied:

(1) $\sum_{k=1}^{\infty}\left(\left\|\Sigma_{k}\right\|_{+}-1\right)$ converges,

(2) $\sum_{k=1}^{\infty}\left(1-\left\|\Sigma_{k}\right\|_{-}\right)$diverges.

Proof. Thereby establishing these two conditions, from Theorem 4, it is obtained that $\prod_{k=1}^{\infty} \Sigma_{i_{k}}=0$. From Corollary 6, Lemma 2 , and by Definition $1,\left\|\Sigma_{k}\right\| \leq \gamma, \gamma<1$, for all $\Sigma_{k} \in \Sigma$. Therefore, according to Theorem $5, \widehat{\rho}(\Sigma)<1$, and so the system (6) is GUAS.

Corollary 9. Let $\left(\Sigma_{k}\right)_{k \in \mathbb{N}}$ be a sequence of switching systems of the form (16) with $\left\|A_{i}\right\| \leq \alpha<1,\left\|C_{i}\right\| \leq \gamma<1$. The discrete

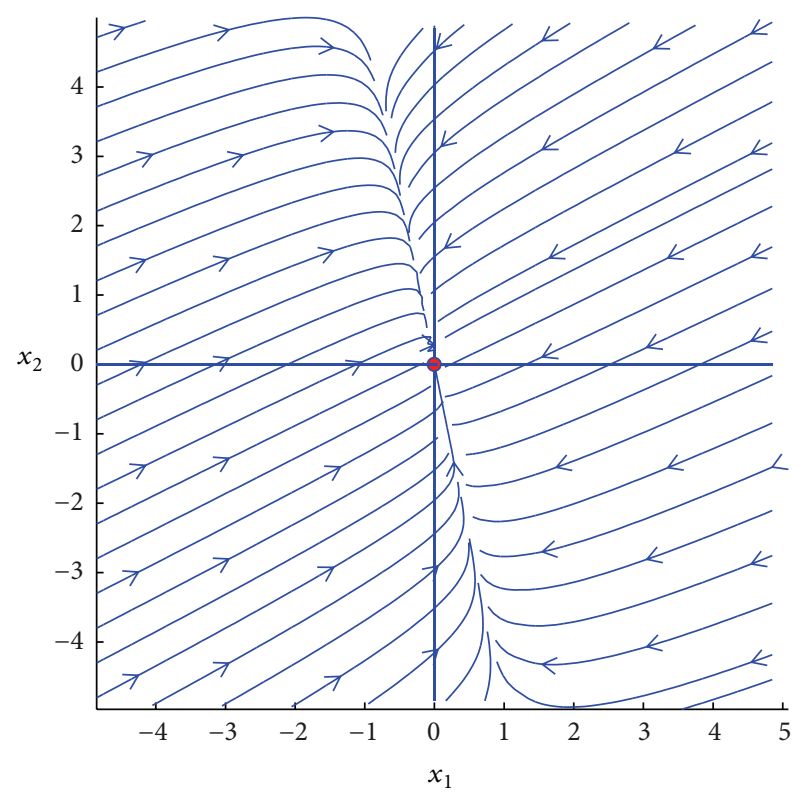

FIgURE 1: Phase portrait of switching linear system with two subsystems.

time switching linear system (6) with an infinite number of switching system is GUAS under arbitrary switching if

$$
\lim _{k \rightarrow \infty} A_{1} A_{2} \cdots A_{k-1} B_{k}\left(I-C_{k}\right)^{-1}=0 .
$$

Arbitrary switching refers to switching systems that there are no restrictions on the discrete event dynamics.

Proof. By Definition 1 and Theorem 7, any infinite product of this kind of switching linear system under these conditions converges to 0 . By definition of the joint spectral radius mentioned in (5),

$$
\begin{aligned}
\rho(\Sigma) & =\lim _{k \rightarrow \infty} \rho_{k}(\Sigma) \\
& =\lim \max \left\{\left\|A_{i_{1}} A_{i_{2}} \cdots A_{i_{k}}\right\|^{1 / k}: i_{j} \in\{0,1, \ldots, n\}\right\},
\end{aligned}
$$

and because of $\left\|A_{i}\right\| \leq \alpha<1$,

$$
\left\|\prod_{j=1}^{k} A_{i_{j}}\right\|^{1 / k} \leq \prod_{j=1}^{k}\left\|A_{i_{j}}\right\|^{1 / k} \leq \alpha^{k}<1, \quad \forall k .
$$

Then,

$$
\rho_{k}(\Sigma) \leq \alpha<1 \Longrightarrow \lim _{k \rightarrow \infty} \rho_{k}(\Sigma) \leq \alpha<1 .
$$

So, $\rho(\Sigma)<1$ and the switching linear system is GUAS.

Lemma 10. Consider the discrete-time switching linear system (6) with matrix structure of the form (16) and $B_{k}=0$. The switching system is GUAS if and only if $\left\{A_{1}, A_{2}, \ldots, A_{k}, \ldots\right\}$, $\left\{C_{1}, C_{2}, \ldots, C_{k}, \ldots\right\}$ are GUAS. 


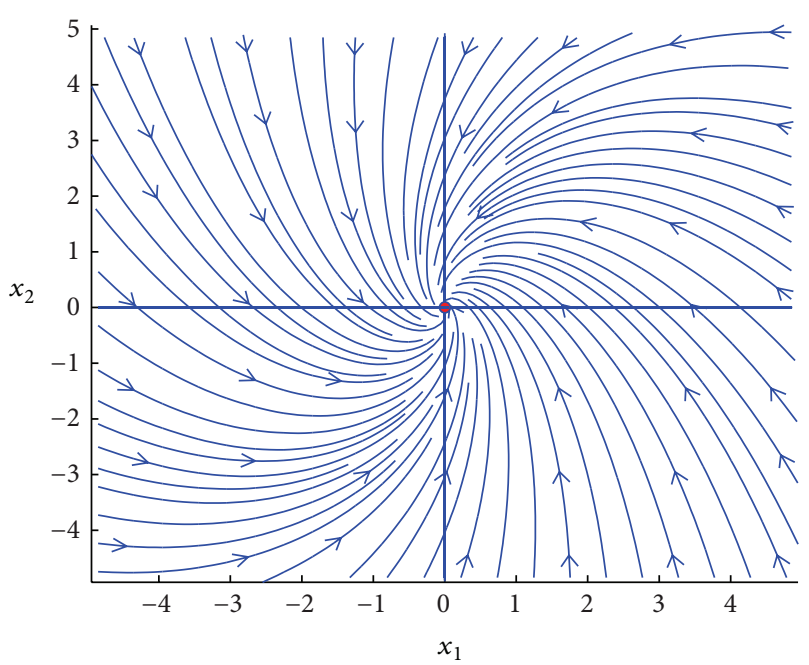

(a) $k=10$

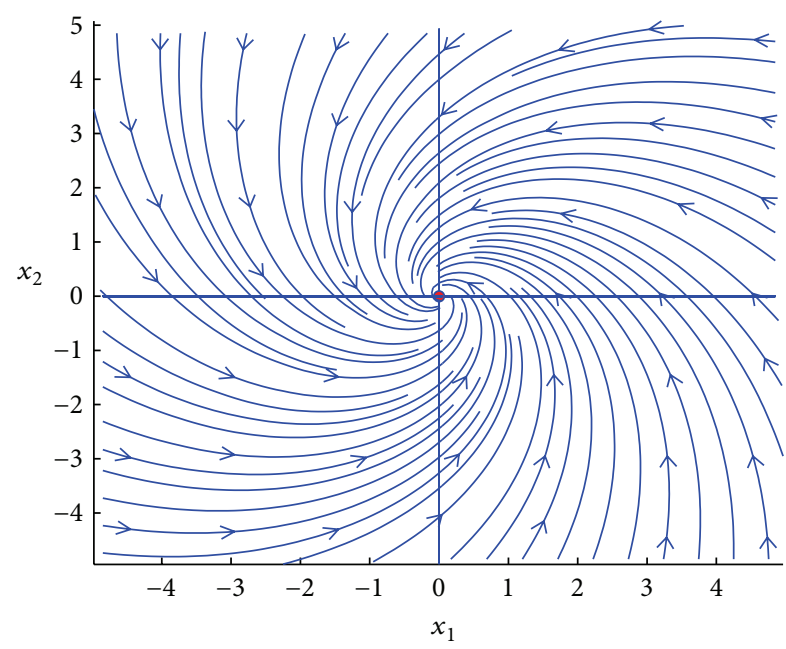

(c) $k=1000$

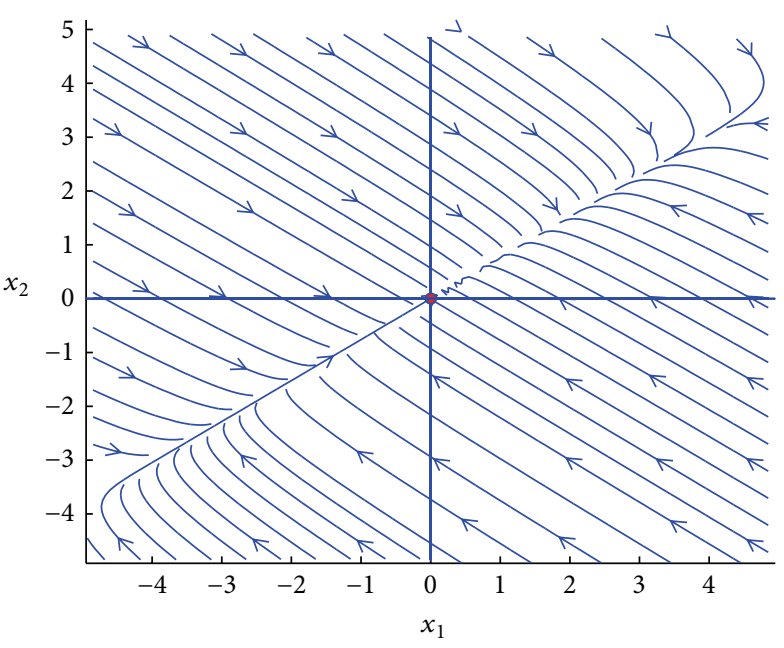

(b) $k=100$

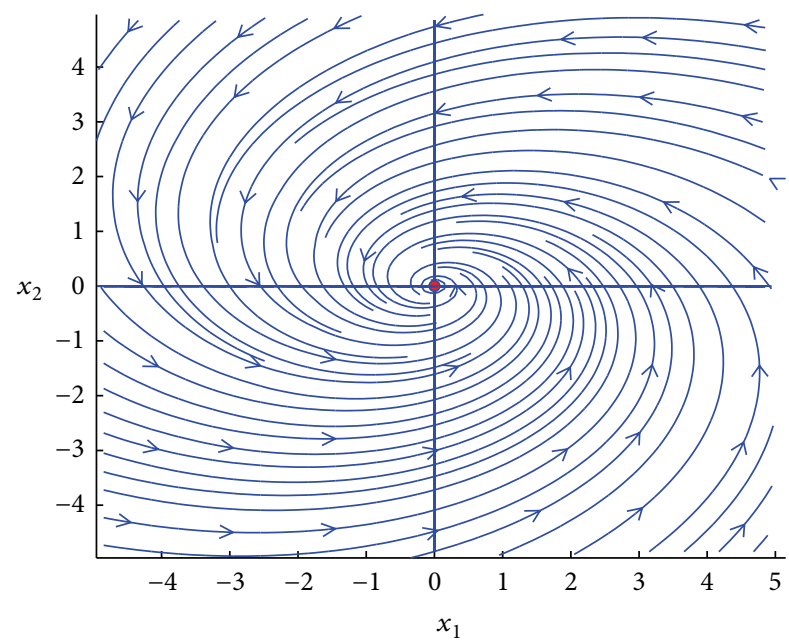

(d) $k=2000$

FIgURE 2: Phase portrait and switching surfaces with 10, 100, 1000, and 2000 subsystems.

Proof. Let $\left\{\Sigma_{k}\right\}$ of the form $\left[\begin{array}{cc}A_{k} & 0 \\ 0 & C_{k}\end{array}\right]$ where $\left[A_{k}\right] \in R^{n_{1} \times n_{1}}$ and $\left[C_{k}\right] \in R^{n_{2} \times n_{2}}$.

Then,

$$
P_{k}=\Sigma_{1} \Sigma_{2} \cdots \Sigma_{k}=\left[\begin{array}{cc}
A_{1} A_{2} \cdots A_{k} & 0 \\
0 & C_{1} C_{2} \cdots C_{k}
\end{array}\right] \text {, }
$$

such that the state $x \in \mathbb{R}^{n}, \Sigma_{k} \in \mathbb{R}^{n \times n}$. So

$$
\begin{aligned}
& \lim _{k \rightarrow \infty} P_{k} \rightarrow 0 \text { if and only if, } \\
& \lim _{k \rightarrow \infty} A_{1} A_{2} \cdots A_{k} \rightarrow 0, \lim _{k \rightarrow \infty} C_{1} C_{2} \cdots C_{k} \rightarrow \\
& 0 .
\end{aligned}
$$

In fact, the discrete-time switching linear system (6) with $B_{k}=0$ is GUAS if and only if $\left\{A_{1}, A_{2}, \ldots, A_{k}, \ldots\right\}$, $\left\{C_{1}, C_{2}, \ldots, C_{k}, \ldots\right\}$ are GUAS.

Result 1. The discrete time switching linear system (6) with an infinite number of switching systems of the form (16) is
GUAS under arbitrary switching if there exist two numbers $\alpha$ and $\gamma$ such that, $\left\|A_{i}\right\| \leq \alpha<1,\left\|C_{i}\right\| \leq \gamma<1$, and $B_{i} \leq \beta<\infty$ is bounded.

Result 2. Because of $\rho(\cdot) \leq\|\cdot\|$, the discrete time switching linear system (6) with an infinite number of switching systems with the form (16) is GUAS under arbitrary switching if $\rho\left(A_{i}\right)<1, \rho\left(C_{i}\right)<1$.

Example 11. Consider switching linear system (6) with two subsystems of the form (16) and let $\Sigma$ be a set of triangular block matrices with blocks on the diagonal:

$$
\Sigma=\left\{\Sigma_{1}, \Sigma_{2}\right\}=\left\{\left[\begin{array}{ccc}
-.1 & .3 & 5 \\
-.5 & .1 & 2 \\
0 & 0 & .8
\end{array}\right],\left[\begin{array}{ccc}
-.4 & -.2 & 4 \\
-.5 & .3 & 5 \\
0 & 0 & .2
\end{array}\right]\right\} .
$$

So

$$
\Sigma_{k}=\left[\begin{array}{cc}
A_{k} & B_{k} \\
0 & C_{k}
\end{array}\right], \quad k=1,2 .
$$


Because of

$$
\begin{gathered}
\left\|A_{1}\right\|=\left\|\begin{array}{cc}
-.1 & .3 \\
-.5 & .1
\end{array}\right\|=0.5414<1, \\
\left\|A_{2}\right\|=\left\|\begin{array}{cc}
-.4 & -.2 \\
-.5 & .3
\end{array}\right\|=0.6531<1, \\
\left\|C_{1}\right\|=0.8<1, \quad\left\|C_{2}\right\|=0.2<1, \\
\left\|B_{1}\right\|=\left\|\begin{array}{l}
5 \| \\
2
\end{array}<\infty, \quad\right\| B_{2}\|=\| \begin{array}{l}
4 \\
5
\end{array} \|<\infty,
\end{gathered}
$$

the switching linear system under arbitrary switching is GUAS.

Figure 1 shows the simulation result of Example 11.

Example 12. Consider a discrete time switching linear system of the form (16) that $\Sigma=\left\{\Sigma_{1}, \Sigma_{2}, \ldots\right\}$, and

$$
\Sigma_{k}=\left[\begin{array}{ccc}
\frac{1}{3 k} & \cos (k) & 4 \\
.5 & \frac{1}{4 k} & 3 \sin (k) \\
0 & 0 & \frac{1}{2 k}
\end{array}\right], \quad k=1,2, \ldots
$$

According to Result 2 of this paper, this switching system with infinite number of subsystems is GUAS under arbitrary switching because of

$$
\begin{gathered}
\rho\left(A_{k}\right)=\rho\left(\left[\begin{array}{cc}
\frac{1}{3 k} & \cos (k) \\
.5 & \frac{1}{4 k}
\end{array}\right]\right)<1, \\
\rho\left(\frac{1}{2 k}\right)<1, \quad \text { for } k=1,2, \ldots
\end{gathered}
$$

So $\lim _{k \rightarrow \infty} A_{1} A_{2} \cdots A_{k-1} B_{k}\left(I-C_{k}\right)^{-1}=0$ for all $k \in \mathbb{N}$. Figure 2 shows some simulation results for $k=10,100,1000$, and 2000 subsystems that switch in a switching system.

\section{Conclusion}

This paper proposed a new necessary and sufficient condition for convergence to zero of infinite product of matrices. Using the conditions to convergence to zero of matrices, stability of switching linear system with an infinite number of subsystems was investigated. Some sufficient conditions were extracted for globally uniformly asymptotically stability (GUAS) of the discrete time switching linear systems.

\section{Conflict of Interests}

The authors declare that there is no conflict of interests regarding the publication of this paper.

\section{References}

[1] H. Lin and P. J. Antsaklis, "Stability and stabilizability of switched linear systems: a survey of recent results," IEEE Transactions on Automatic Control, vol. 54, no. 2, pp. 308-322, 2009.

[2] D. Cheban and C. Mammana, "Absolute asymptotic stability of discrete linear inclusions," Buletinul Academiei de Știinţe a Republicii Moldova: Matematica, no. 1, pp. 43-68, 2005.

[3] M. A. Muller and D. Liberzon, "State-norm estimators for switched nonlinear systems under average dwell-time," in Proceedings of the 49th IEEE Conference on Decision and Control, pp. 1275-1280, December 2010.

[4] D. Liberzon and S. Trenn, "On stability of linear switched differential algebraic equations," in Proceedings of the 48th IEEE Conference on Decision and Control, pp. 2156-2161, December 2009.

[5] A. A. Agrachev, Y. Baryshnikov, and D. Liberzon, "Towards robust Lie-algebraic stability conditions for switched linear systems," in Proceedings of the 49th IEEE Conference on Decision and Control, pp. 408-413, December 2010.

[6] L. V. Hien, Q. P. Ha, and V. N. Phat, "Stability and stabilization of switched linear dynamic systems with time delay and uncertainties," Applied Mathematics and Computation, vol. 210, no. 1, pp. 223-231, 2009.

[7] J. Raouf and H. Michalska, "Exponential stabilization of switched linear systems," in Proceedings of the 48th IEEE Conference on Decision and Control, pp. 1926-1931, December 2009.

[8] H. Xu and K. L. Teo, "Robust stabilization of uncertain impulsive switched systems with delayed control," Computers \& Mathematics with Applications, vol. 56, no. 1, pp. 63-70, 2008.

[9] F. M. Hante and M. Sigalotti, "Converse Lyapunov theorems for switched systems in Banach and Hilbert spaces," SIAM Journal on Control and Optimization, vol. 49, no. 2, pp. 752-770, 2011.

[10] H. Du, X. Lin, and S. Li, "Finite-time stability and stabilization of switched linear systems," in Proceedings of the 48th IEEE Conference on Decision and Control, pp. 1938-1943, December 2009.

[11] G. Zhai, H. Lin, X. Xu, J. Imae, and T. Kobayashi, "Analysis of switched normal discrete-time systems," Nonlinear Analysis: Theory, Methods \& Applications, vol. 66, no. 8, pp. 1788-1799, 2007.

[12] H. Xu and K. L. Teo, "Exponential stability with $L_{2}$-gain condition of nonlinear impulsive switched systems," IEEE Transactions on Automatic Control, vol. 55, no. 10, pp. 24292433, 2010

[13] K. R. Santarelli, "A switched state feedback law for the stabilization of LTI systems," in Proceedings of the American Control Conference, pp. 1701-1707, Baltimore, Md, USA, 2010.

[14] G. Zhai and X. Xu, "A unified approach to stability analysis of switched linear descriptor systems under arbitrary switching," International Journal of Applied Mathematics and Computer Science, vol. 20, no. 2, pp. 249-259, 2010.

[15] Y. Zhang, Y. Zhao, H. Xu, H. Shi, and K. L. Teo, "On boundedness and attractiveness of nonlinear switched delay systems," Abstract and Applied Analysis, vol. 2013, Article ID 713847, 8 pages, 2013.

[16] L. F. Araghi and H. S. Hosseini, "Robust atability of Fuzzy Elman neural network," in Proceedings of the IEEE International Conference on Granular Computing (GRC '09), pp. 13-18, 2009. 
[17] R. C. Loxton, K. L. Teo, and V. Rehbock, "Computational method for a class of switched system optimal control problems," IEEE Transactions on Automatic Control, vol. 54, no. 10, pp. 2455-2460, 2009.

[18] C. Yu, B. Li, R. Loxton, and K. L. Teo, "Optimal discrete-valued control computation," Journal of Global Optimization, vol. 56, no. 2, pp. 503-518, 2013.

[19] S. F. Woon, V. Rehbock, and R. Loxton, "Towards global solutions of optimal discrete-valued control problems," Optimal Control Applications \& Methods, vol. 33, no. 5, pp. 576-594, 2012.

[20] Q. Lin, R. Loxton, and K. L. Teo, "Optimal control of nonlinear switched systems: computational methods and applications," Journal of the Operations Research Society of China, vol. 1, no. 3, pp. 275-311, 2013.

[21] A. A. Suratgar and S. K. Y. Nikravesh, "A new sufficient conditions for stability of fuzzy systems," in Proceedings of the International Conference on Electrical Engineering (ICEE '02), Tabriz, Iran, 2002.

[22] A. A. Suratgar and S. K. Y. Nikravesh, "A new sufficient conditions for asymptotic stability of a class of applied nonlinear dynamical system," in Proceedings of the 10th IEEE International Conference on Electronic, Circuit and Systems, vol. 3, pp. 10621065, 2003.

[23] R. M. Jungers, Infinite matrix products from the joint spectral radius to combinatorics [Ph.D. thesis], 2008.

[24] R. Jungers, The Joint Spectral Radius: Theory and Applications, vol. 385, Springer, Berlin, Germany, 2009.

[25] T. Monovich and M. Margaliot, "A second-order maximum principle for discrete-time bilinear control systems with applications to discrete-time linear switched systems," Automatica, vol. 47, no. 7, pp. 1489-1495, 2011.

[26] A. A. Ahmadi, R. M. Jungers, P. Parrilo, and M. Roozbehani, "Analysis of the joint spectral radius via lyapunov functions on path-complete graphs," in Proceedings of the International Conference on Hybrid Systems: Computation and Control (HSCC '11), Chicago, Ill, USA, 2011.

[27] D. J. Hartfiel, Nonhomogeneous Matrix Products, Birkhäauser, Boston, Mass, USA, 2002.

[28] J. B. Conwey, A Course in Functional Analysis, Springer, 2nd edition, 1997. 


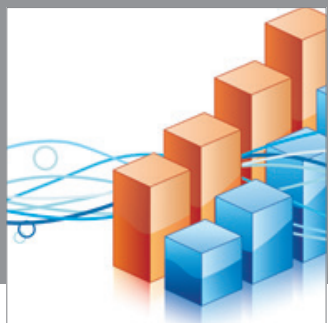

Advances in

Operations Research

mansans

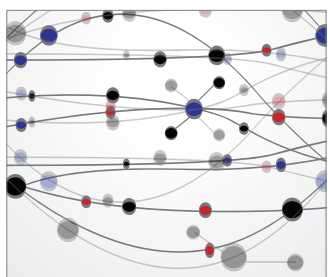

The Scientific World Journal
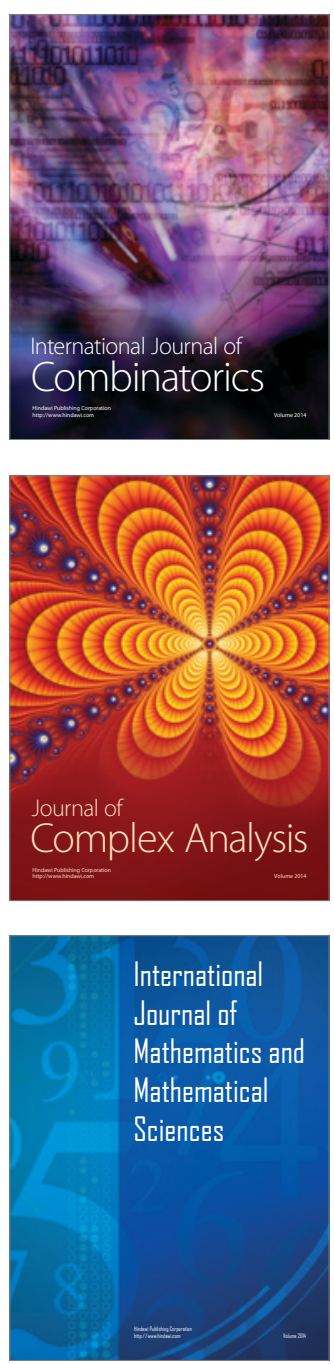
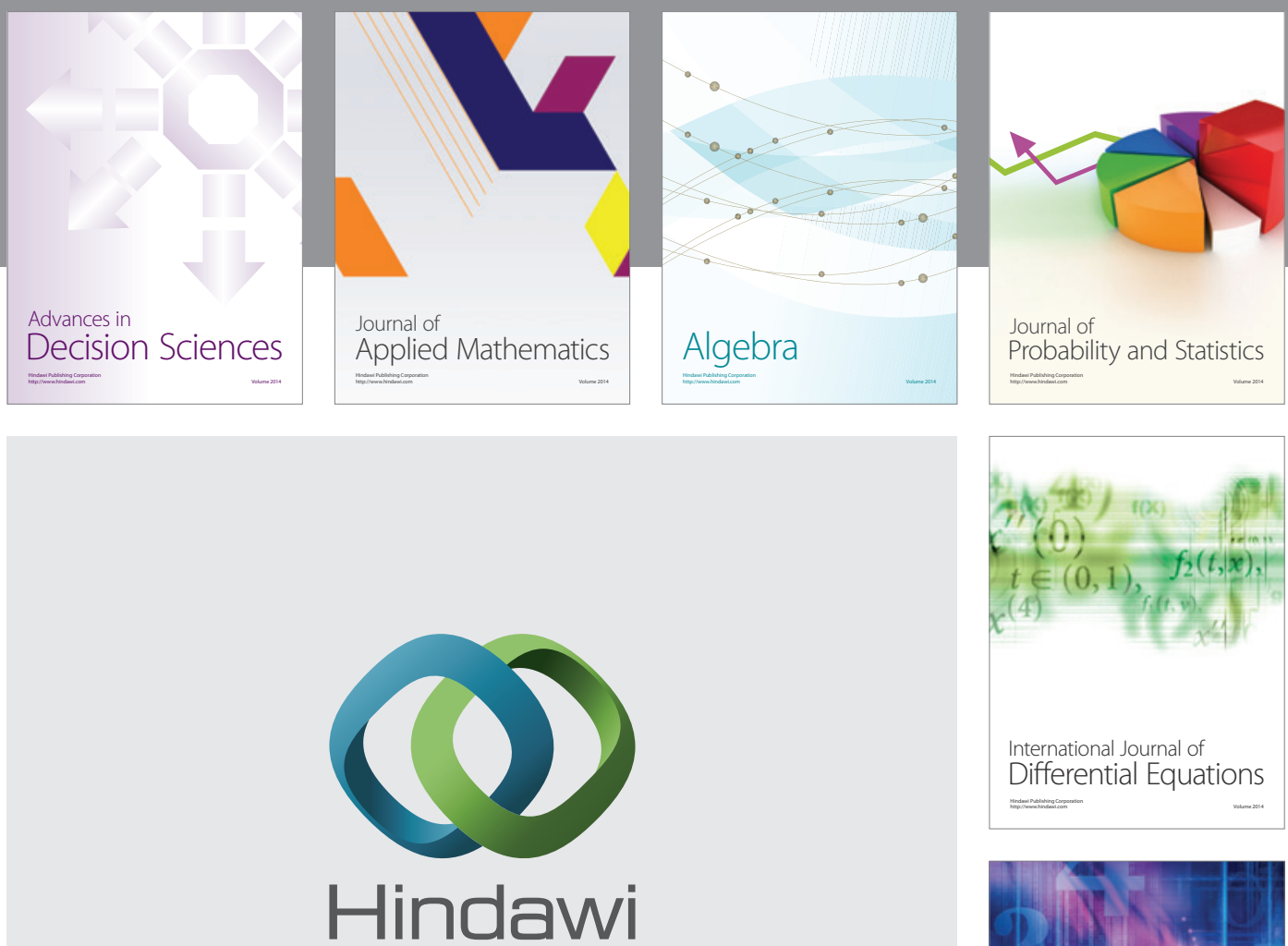

Submit your manuscripts at http://www.hindawi.com
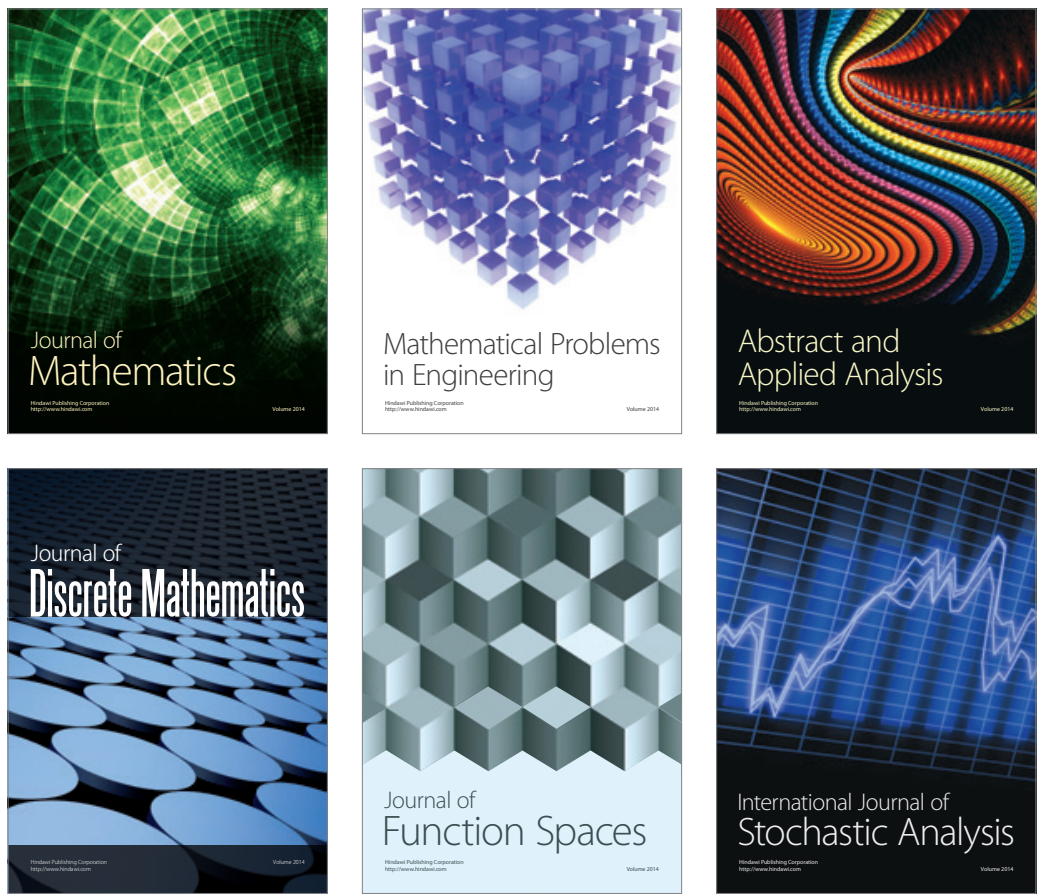

Journal of

Function Spaces

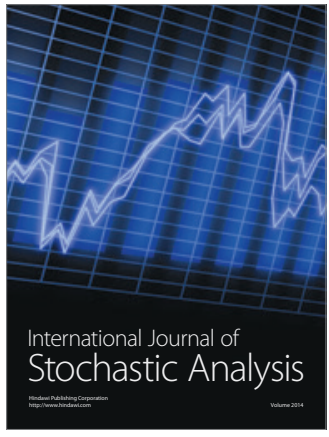

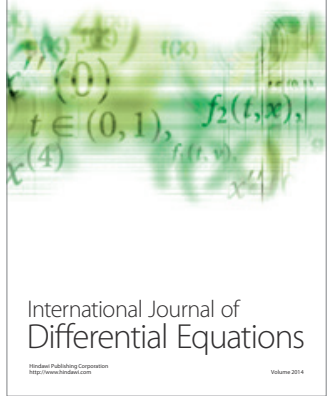
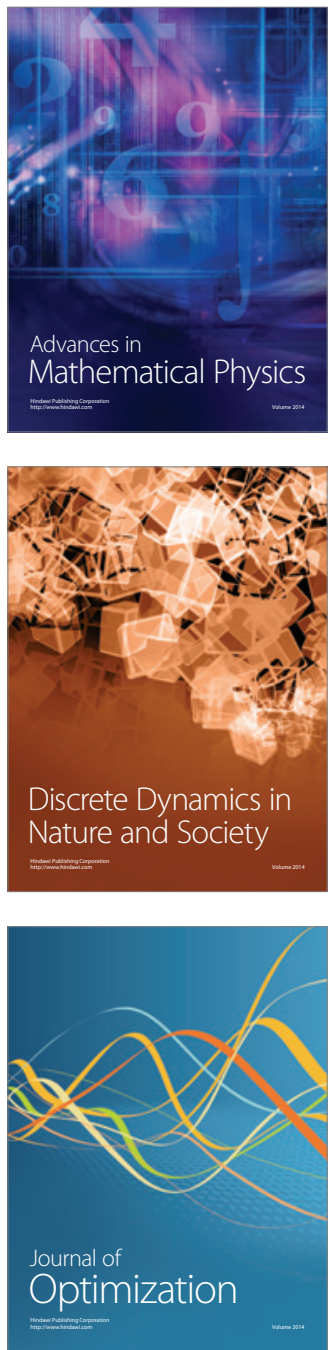